\title{
Response letter
}

Manuscript No. TLCT-2015-0112

Title : Homogeneous alignment of liquid crystals on low temperature solution-derived gallium oxide films via IB irradiation method

Authors : Sang Bok Jang, ${ }^{1}$ Gi-Seok Heo, ${ }^{2}$ Eun-Mi Kim, ${ }^{2}$ Hong-Gyu Park,,${ }^{1}$ Ju Hwan Lee, ${ }^{1}$ Yoon Ho Jung, ${ }^{1}$ HaeChang Jeong, ${ }^{1}$ Jeong-Min Han, ${ }^{3}$ and Dae-Shik Seo ${ }^{1}, *$

We deeply appreciate the reviewer's valuable questions and comments. As the reviewers raised important points for further clarification, we have revised the manuscript in accordance. The modifications and some supplementary comments can be detailed as follows:

1) Authors report that only thermal annealing at $100 \mathrm{C}$ creates $\mathrm{GaO}$ layer that aligns $\mathrm{LC}$. Why GaO layer annealed at higher temperatures doesn't produce good alignment? What happens to $\mathrm{GaO}$ if layer annealed at higher temperatures? Authors briefly should comment on this.

We duplicated the experiment for LC alignment characteristics with respect to annealing temperature, and same results were obtained. However, we did not proceed further analyses because the random LC alignments on the $\mathrm{GaO}$ films were meaningless in the LCD applications. We have conducted experiments for LC alignment characteristics on the $\mathrm{GaO}$ films with respect to intensity of IB energy instead of in-depth analyses for random LC alignment on the $\mathrm{GaO}$ films annealed at high temperature.

2) Besides transmittances of $\mathrm{GaO}$ films annealed at different temperature Figure 2 should show transmittances of ITO glass and PI coated ITO glass which authors mention in the text. 
As the reviewer pointed out, we corrected Fig. 1.

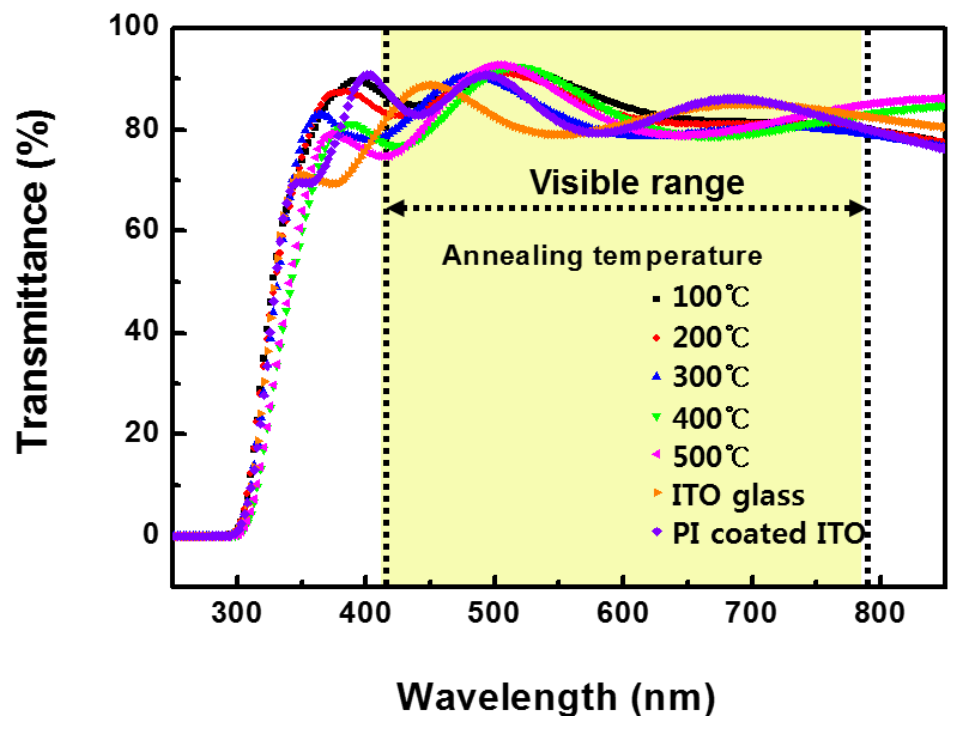

3) Authors state that for annealing at $100 \mathrm{C}$ "Uniformly aligned LCs were observed on the surfaces of the IB irradiated GaO films regardless of the intensity of IB energy". How does LC align on GaO surface which was not irradiated with IB? It is understood that IB irradiation is needed to generate the alignment. Therefore, one may expect there should be a minimum energy of IB below which alignment would not be generated. Such energy threshold for IB is an essential detail to discuss. What is the reason why authors didn't try IB energies less than $600 \mathrm{eV}$ ?

As shown in Fig. S1, random LC alignment was obtained on the non-IB-irradiated GaO film. Specifically, the photomicrograph of LC cell based on non-IB-irradiated $\mathrm{GaO}$ film shows that $\mathrm{LC}$ molecules on the pristine $\mathrm{GaO}$ film appears to be partially aligned. However, a high error value was measured in the LC cell based on non-IBirradiated $\mathrm{GaO}$ film through TBA 107 transmittance curve for the measurement of the pretilt angle, resulting in the random LC alignment.

Taking all those things together, even low intensity of IB energy is required to achieve the uniform LC alignment. The reason for not using IB intensity below $600 \mathrm{eV}$ is because it is difficult to secure reliability at low IB intensity. 

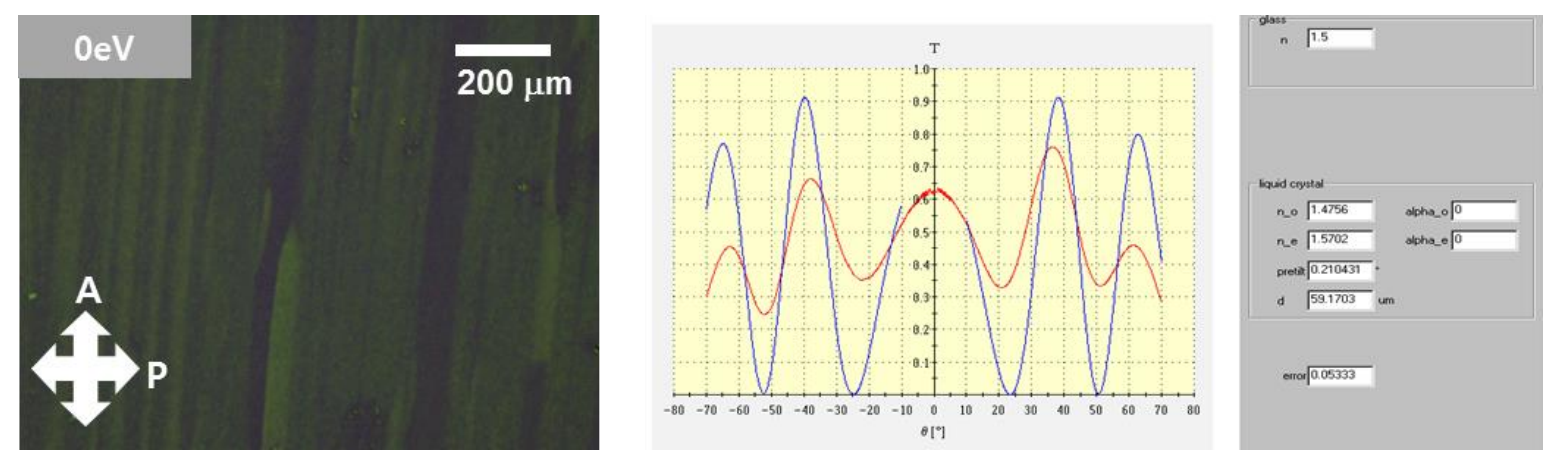

Figure S1. Photomicrographs of anti-parallel cell with non-IB-irradiated $\mathrm{GaO}$ films and transmittance versus incident angle of the LC molecules on the non-IB-irradiated $\mathrm{GaO}$ films.

- In the revised manuscript:

While random LC alignment was observed on the non-IB-irradiated $\mathrm{GaO}$ film, uniformly aligned LCs were observed on the surfaces of the IB-irradiated $\mathrm{GaO}$ films regardless of the intensity of IB energy. Partially distinguishable line defects were observed in the POM images; however, these defects were invisible to the naked eye. Even though the IB irradiation of low intensity, the increased anisotropy of the GaO films by IB irradiation induced uniform LC alignment.

4) Reported values of measured pretilt angle are not consistent with the change of IB energy. Can authors comment on this behavior? Is there any physics behind it? What is the precision of pretilt angle measurement setup used to obtain those values?

- The pretilt angles of LC molecules on the $\mathrm{GaO}$ films were measured by the TBA 107 measurement. As shown in Fig. S1, if the measured (blue line) and simulated (red line) curves are identical, then the LC alignment is uniform and the pretilt angle of the LC molecules can be accurately estimated. The measurement range of the pretilt angles are from 0 (homogeneous LC alignment) to $90^{\circ}$ (vertical LC alignment). Therefore, the pretilt angles range from 0.01 to $0.2^{\circ}$ of LC molecules is almost the same level. The difference is within the margin of error.

5) Plot shown on Fig. 3(b) is difficult to read since values of pretilt angle at different data points are not distinguishable. The figure 3(b) has to be revised or removed. 
In the manuscript, we revised Fig. 3(b).

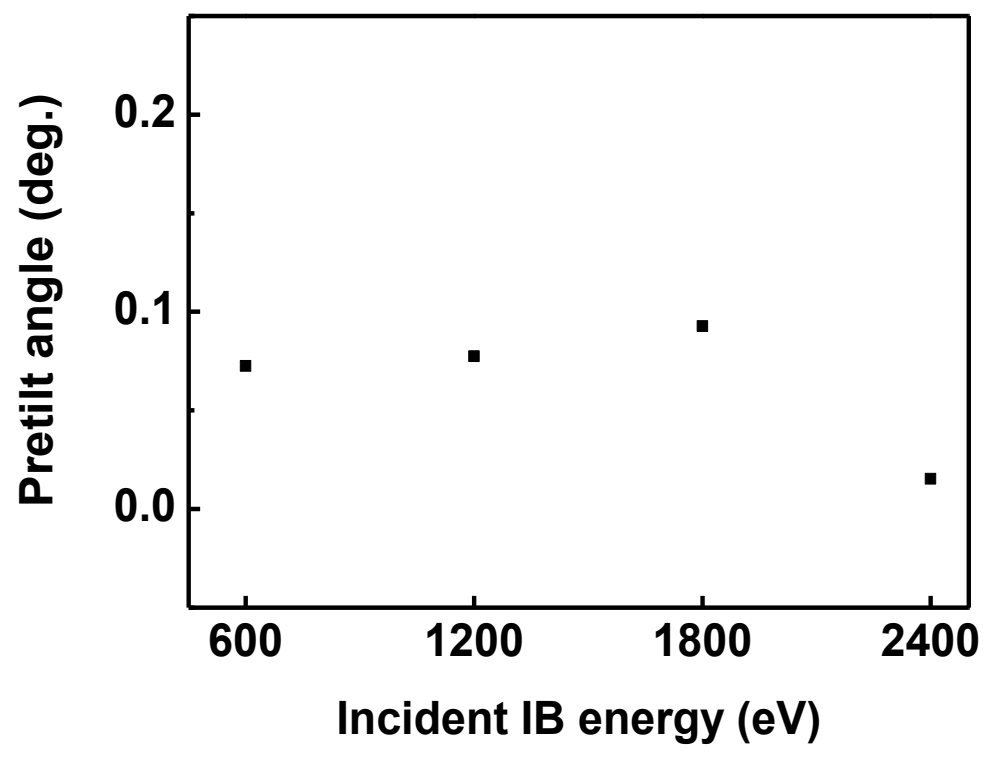

6) I don't see the relationship of reference [36] to sentence: "As shown in Fig. 6(a), the threshold voltages of the TN cells fabricated with $600 \mathrm{eV}, 1200 \mathrm{eV}, 1800 \mathrm{eV}$, or $2400 \mathrm{eV}$ IB-irradiated GaO films and a rubbed PI layer were 1.837 V, 1.661 V, 2.124 V, $1.58 \mathrm{eV}$, and 2.01 V [36], respectively." Also, "1.58 eV" seems to be “1.58 V". Some numbers are reported with two and some with three significant figures after decimal point. This should be consistent throughout the paper. Authors should comment why values of reported threshold voltages look random with respect to IB energy. This may be partially from improper interpretation of data shown on Fig. 6(a).

Done.

[Results and Discussion]

As shown in Fig. 7(a), the threshold voltages of the TN cells fabricated with $600 \mathrm{eV}, 1200 \mathrm{eV}, 1800 \mathrm{eV}$, or 2400 eV IB-irradiated $\mathrm{GaO}$ films and a rubbed PI layer were 1.578 V, 1.661 V, 2.124 V, 1.580 V, and 2.010 V [36], respectively. 
Reference [36] is only added to quote the data for the rubbed PI.

Additionally, typo was corrected. We miswrote a threshold voltage value of TN cell at IB intensity of $600 \mathrm{eV}$. $1.837 \mathrm{~V} \rightarrow 1.578 \mathrm{~V}$

7) What voltages cells are being switched between to get data shown on Fig. 6(b)?

According to the response time, the rise time and fall time are defined as $90 \rightarrow 10 \%$ and $10 \rightarrow 90 \%$ transmittance change, respectively. In the LCMS-200 measurement, rise time and fall time of TN cells were separately measured as shown in below example figure.

The voltages at $90 \%$ and $10 \%$ transmittance were obtained from V-T curves of TN cells using an LCMS-200 measurement.

\begin{tabular}{|c|c|c|}
\hline \multirow{2}{*}{ IB incident energy } & \multicolumn{2}{|c|}{ Voltage at \% transmittance } \\
\cline { 2 - 3 } & V90 & V10 \\
\hline $600 \mathrm{eV}$ & 1.578 & 3.492 \\
\hline $1200 \mathrm{eV}$ & 1.661 & 3.673 \\
\hline $1800 \mathrm{eV}$ & 2.124 & 3.827 \\
\hline $2400 \mathrm{eV}$ & 1.580 & 4.132 \\
\hline
\end{tabular}

\section{[For example]}

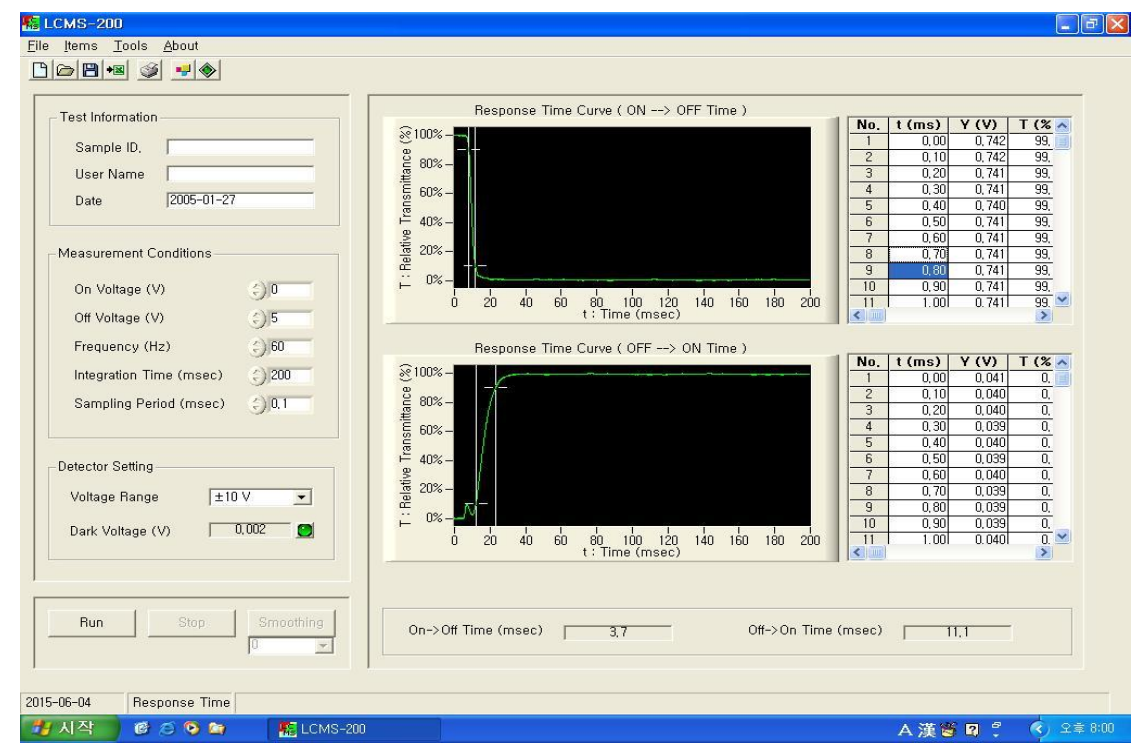




\section{8) Reference [18] is numbered not in order.}

- We renumbered references correctly.

\section{[Introduction]}

Strategies to overcome the weaknesses of the rubbing method have included the ultraviolet (UV) photoalignment technique [12,13], oblique deposition [14,15], nanoimprint lithography [16,17], and ion-beam (IB) irradiation [1822]. Among these, collimated IB irradiation is a promising non-contact alignment method that uses Ar+ ioninduced plasma to furnish controllability in the manufacture of high-resolution displays in an uninterrupted process. In addition, the IB technique can induce a unidirectional orientation of LC molecules on both organic and inorganic materials. Due to these merits, the IB technique has been intensively investigated with optically transparent insulating inorganic materials, such as diamond-like carbon [19,23], SnO2 [21], SiOx [24], SiNx [25], and Ta2O5 [26], as well as a variety of organic PI materials [27].

9) Are there any references to support the last part of sentence: "Due to these merits, the IB technique has been intensively investigated with optically transparent insulating inorganic materials, such as diamondlike carbon [18,19], $\mathrm{SnO}$ [21], $\mathrm{SiOx}$ [24], $\mathrm{SiNx}$ [25], and Ta2O5 [26], as well as a variety of organic PI materials."

In agreeing with the reviewer, we added an additional reference.

[Introduction]

Due to these merits, the IB technique has been intensively investigated with optically transparent insulating inorganic materials, such as diamond-like carbon [19,23], SnO2 [21], SiOx [24], SiNx [25], and Ta2O5 [26], as well as a variety of organic PI materials [27]. 
[references]

[27] Oh B-Y, Lee K-M, Kim B-Y, Kim Y-H, Han J-W, Han J-M, Lee S-K, Seo D-S. Surface reformation and electro-optical characteristics of liquid crystal alignment layers using ion beam irradiation. J Appl Phys. 2008;104:064052.

\section{Additional modifications:}

1) Additional authors (Eun-Mi Kim and Jeong-Min Han) were included.

Thank you very much for your remarks again!

Dae-Shik Seo

Professor 